\title{
GENERATIONS OF COMPUTER
}

\author{
Nonika Sharma ${ }^{1 *}$, Rashika Kaul ${ }^{2}$ \\ *1,2 IT Department Dronacharya College of Engg. Gurgaon,Haryana
}

*Corresponding Author: -

\begin{abstract}
: -
In our research paper we are going to focus on $2 G, 3 G, 4 G$ generation systems and their standards. Also, we will focus on the various advantages and disadvantages of each generation system. We will also study about the various technologies used in each generation system.
\end{abstract}

\section{(c) $(\$)$}




\section{INTRODUCTION}

$2 \mathrm{G}$ uses cellular technology. It began using digital signaling whereas $3 \mathrm{G}$ is the generation of wireless technology. It can transfer both voice and non-voice data. 4G uses SDR and it is based only on a packet switching network.

\section{G (Second Generation)}

It uses digital signaling. Depending upon the time of multiplexing used $2 \mathrm{G}$ is categorized into CDMA and TDMA based. The main $2 \mathrm{G}$ standards are-
1. GSM
2. DEN
3. $\mathrm{PDC}$

\section{ADVANTAGES OF 2G}

1. It aided in digital error checking, reducing noise.

2. Digital data services were introduced

\section{DISADVANTAGES OF 2G}

1. Slow speed

2. Low power emission

\section{G (THIRD GENERATION)}

It is the generation of wireless technology and offers high data transfer rates for handheld devices. It also provides some multimedia services that are combination of both voice and data.

\section{FEATURES OF 3G}

1. It supports multimedia

2. Connectivity is always up

3. It supports quick download of large files

\section{STANDARDS OF 3G}

CDMA 2000, WCDMA, FDMA/TDMA

\section{ADVANTAGES}

1. It provides both fixed and variable data rates.

2. It provides security.

3. It provides reliability.

4. Data rates are symmetric.

\section{DISADVANTAGES}

1. Cost is high

2. Power requirement is high.

\section{APPLICATIONS OF 3G}

1. Global Positioning System (GPS)

2. Location-based services

3. Mobile TV

4. Telemedicine

5. Video Conferencing

6. Video on demand

\section{G (FOURTH GENERATION)}

4G is based only on packet switching, which helps in low latency data. $4 \mathrm{G}$ allows smooth video transmission with high quality and is 1020 times faster than ADSL services. 4G system, in addition to the usual voice and other services of 3G, provides mobile broadband Internet access, for example to laptops with wireless modems, to smartphones, and to other mobile devices. Potential and current applications include amended mobile web access, IP telephony, gaming services, high-definition mobile TV, video conferencing, 3D television, and cloud computing.
ADVANTAGES
1. It uses SDR.
2. It allows smooth video transmission.

\section{Open-wireless Architecture and Software-defined radio (SDR)}

One of the key technologies for $4 \mathrm{G}$ and beyond is called Open Wireless Architecture, supporting multiple wireless air interfaces in an open architecture platform. 
SDR is one form of open wireless architecture (OWA). Since 4G is a collection of wireless standards, the final form of a 4G device will constitute various standards. This can be efficiently realized using SDR technology, which is categorized to the area of the radio convergence.

\section{HISTORY OF 4G}

The 4G system was originally envisioned by the Defense Advanced Research Projects Agency .The DARPA selected the distributed architecture and end-to-end Internet protocol (IP), believed at an early stage in peer-to-peer networking in which every mobile device would be both a transceiver and a router for other devices in the network, eliminating the spoke-and-hub weakness of $2 \mathrm{G}$ and $3 \mathrm{G}$ cellular systems. Since the $2.5 \mathrm{G}$ GPRS system, cellular systems have provided dual infrastructures: packet switched nodes for data services, and circuit switched nodes for voice calls. In $4 \mathrm{G}$ systems, the circuit-switched infrastructure is abandoned and only a packet-switched network is provided, while $2.5 \mathrm{G}$ and $3 \mathrm{G}$ systems require both packet-switched and circuit-switched network nodes, i.e. two infrastructures in parallel. 